AperTO - Archivio Istituzionale Open Access dell'Università di Torino

\title{
Evolutionary minority games with memory
}

\section{This is the author's manuscript}

Original Citation:

Availability:

This version is available http://hdl.handle.net/2318/1652614

since 2017-11-22T11:27:17Z

Published version:

DOI:10.1007/s00191-017-0526-4

Terms of use:

Open Access

Anyone can freely access the full text of works made available as "Open Access". Works made available under a Creative Commons license can be used according to the terms and conditions of said license. Use of all other works requires consent of the right holder (author or publisher) if not exempted from copyright protection by the applicable law. 


\title{
Evolutionary minority games with memory
}

\author{
Gian Italo Bischi \\ Università di Urbino "Carlo Bo" \\ Ugo Merlone \\ Università di Torino
}

\begin{abstract}
We propose a simple dynamic adjustment mechanism, equivalent to the standard replicator dynamics in discrete time, to study the time evolution of a population of players facing a binary choice game, and apply this mechanism to minority games in order to investigate the effects of memory on the stability of the unique Nash equilibrium. Two different kinds of memory are considered, one where the players take into account the current and the previous payoffs in order to decide the strategy chosen in the next period, and the other one where the players consider the whole series of payoffs observed in the past through a discounted sum with exponentially fading weights. Both the memory representations proposed lead to an analytically tractable two-dimensional dynamical system, so that analytical results can be given for the stability of the Nash equilibrium, However, a global analysis of the models performed by numerical methods and guided by the analytical results shows that some complexities arise for intermediate values of the memory parameter, even if the stabilization effect of uniform memory is stated in both cases.
\end{abstract}

JEL Classification: C72, C73.

Keywords: Binary games, Minority games, Replicator dynamics, Memory, Stability.

\section{Introduction}

Collective behavior has been studied by several disciplines such as sociology [21], politics [26], social psychology [2], industrial organization [17], marketing [29], and communication [35] to name a few. In fact, according to 
[20], several processes such as residential segregation, voting, crowd behavior, diffusion of innovations and consumption choices can be considered as situations in which people adopt new norms or abandon existing ones.

Several contributions have examined binary choice games with externalities, i.e. a social system composed by a population of agents facing a binary choice such that the payoff obtained by each agent as a consequence of the option chosen is affected by the number of agents currently making the same choice (see e.g. [31], [32], [21]). In particular, repeated binary choice games, expressed in the form of discrete dynamical systems, have recently been studied by [5], [6], and different kinds of long run behaviors have been evidenced, such as convergence to an equilibrium situation or endless self sustained endogenous oscillations, both periodic and chaotic. When considering how individuals or populations change their strategy over time based on payoff comparisons, it is common to consider the approach of evolutionary games. In particular, according to [12], "the replicator equation is the first and most important game dynamics studied in connection with evolutionary game theory".

In this paper, we propose a dynamic model in discrete time, based on a particular kind of replicator dynamics proposed in [7] and discussed in [24], to describe the time evolution, and, in particular, the long run behavior of a binary choice game. The mathematical model proposed is expressed by an elegant one-dimensional discrete dynamical system that simply states that, at each time step $t$, a fraction of agents will change their choice at time $t+1$ by switching to the option that is observed to give a higher payoff in the current time period. Such an evolutionary model is applied to a well known class of binary games, denoted as minority games, characterized by the property that the players who select the option chosen by the minority are more rewarded. It is well known in the literature, and quite intuitive, that a population of agents that repeatedly play, in discrete time periods, a minority game, tends to exhibit oscillations between the two strategies. In fact, players who choose the majority strategy are oriented to revise their decision towards the option chosen by minority, and as a result, oscillatory behaviors are typically observed. Such oscillations may either converge in the long run to an equilibrium situation - generally a Nash equilibrium characterized by identical payoffs associated to the two choices - or continue indefinitely, endogenously self-sustained by overshooting phenomena, without settling to any stationary equilibrium in the long run (see e.g. [32], [5]). Quite famous are the examples in the paper by Schelling (1973) such as the one concerning 
the binary choice about whether to use the car or not, depending on the traffic congestion, or the celebrated 'El Farol Bar Problem' proposed by Arthur in [3].

Minority games have also been proposed as simple stylized models able to capture the essence of some features of the financial markets [10]. Furthermore, [18] pointed how this situation could be useful as a special case of market entry games.

In order to find adaptive mechanisms that stabilize the oscillations in repeated minority games, several authors have proposed the introduction of memory in the decision process, i.e. the players' decisions are not only based on the current payoffs observed, but also take account of payoffs observed in the past. However, the effects of memory is revealed to be not univocal, and several ambiguous conclusions can be found in the literature. (A comparison of the titles of references [8] and [9] is quite emblematic.)

The motivation of this paper is to modify the evolutionary model proposed by [14] in order to consider how past payoffs can affect current choices in two different ways. The two ways we consider are both based on a weighted average of the past outcomes and lead to a two-dimensional discrete dynamical system characterized by analytical tractability. The first proposed method assumes that the players, in order to decide the next period strategy choice, consider a weighted average, i.e., a convex combination of the current payoffs and those observed in the previous time period (memory of length one). The second method is the same considered in [14], and considers a weighted sum of all previously observed payoffs with exponentially (or geometrically) distributed weights that discount past outcomes. Both these generalization of the evolutionary model without memory are performed in a way that does not modify the equilibrium points of the original model, and only affects their stability properties. Our analytical results, expressed by three propositions concerning local stability of the Nash equilibrium as well as the local bifurcations leading to oscillatory behavior, give threshold values of the memory parameter that depend on the speed of reaction by which the players react to perceived differences in observed payoffs. On the basis of these analytical results on local properties of the dynamic models around the Nash equilibrium, we also perform numerical explorations of global properties of the same models. In this way we are able to examine the long run outcomes obtained starting from initial conditions far from the Nash equilibrium. Furthermore, we can analyze the dynamic effects observed when parameters' constellations are considered with values quite different with re- 
spect to the ones close to the stability ranges according to the proposition proved. These numerical results reveal new, and sometimes unexpected, properties of the evolutionary models with memory, so that some interesting lessons on the methods used to study nonlinear dynamical systems are drawn. In fact, phenomena of multistability, i.e. coexistence of several attracting sets that characterize the long run dynamics, are observed. The presence of these coexisting attractors, each with its own basin of attraction, give rise to a strong path dependence, sometimes called "corridor stability" in the economic literature (see [27]), and cannot be revealed by the analytical study of local stability based on the standard linearization procedure of the dynamical systems.

This paper is organized as follows. Section 2 presents the basic setup of a model of repeated binary game with evolutionary dynamics based on the exponential replicator. Section 3 proposes the generalization of the model by considering a finite memory of length 1, and gives the proposition on the effects of memory on local stability of the Nash equilibrium, as well as the numerical analysis of some global dynamic properties, in particular, the coexistence of attractors. Then, infinite and exponentially discounted memory is introduced and results are proved about the stabilizing effects of memory. Section 4 concludes and indicates further applications of the methods outlined in this paper.

\section{A Model of binary choice with evolutionary dynamics}

Let us consider a population in which agents form unitary continuum. Each agent faces a binary choice between two strategies: $R$ and $L$. At time $t$ let $x(t) \in[0,1]$ be the fraction of agents playing $R$; consequently $1-x(t)$ play $L$ at the same time. Assume that the individual payoff of an agent employing a given strategy at time $t$ depends only on the number of agents making the same choice or the other, say $R(t)=R(x(t))$ and $L(t)=L(x(t))$.

This framework is general enough to include several games such as the well known $n$-players prisoner's dilemma or minority games (e.g. [11]). Schelling $[31,32]$ proposes several vivid and stimulating examples, taken from economics, social sciences and everyday life, which can be represented in this framework.

We now introduce a discrete-time evolutionary process to describe the number of agents that at each time period $t=0,1,2, \ldots$ update their choice, under the assumption that the payoff obtained by both fractions of players at 
time $t$, i.e. $R(x(t))$ and $L(x(t))$, are common knowledge. The time evolution of the fraction $x(t)$ of players choosing $R$ is assumed to be monotonically influenced by the "gain" function

$$
g(x)=R(x)-L(x):[0,1] \rightarrow \mathbb{R}
$$

in the sense that higher gains cause an increase of the fraction of agents choosing $R$. A typical monotone selection dynamics is given by the one proposed in [7]. This dynamics eliminates dominated strategies and is analyzed in [24]; we consider this dynamics as, according to [34], experimental investigations have found that subjects generally avoid dominated strategies.

$$
\begin{aligned}
x(t+1)=f(x(t)) & =\frac{x(t) \exp (\alpha R(x(t)))}{x(t) \exp (\alpha R(x(t)))+(1-x(t)) \exp (\alpha L(x(t)))} \\
& =\frac{x(t)}{x(t)+(1-x(t)) \exp (-\alpha g(x(t)))}
\end{aligned}
$$

where $\alpha>0$ is the speed of reaction, a parameter that expresses the propensity to switch to the opposite choice as a consequence of a payoff gain observed in the current time period. It is worth remarking that, if $x(0) \in[0,1]$, then $x(t) \in[0,1]$ for each $t \geq 0$, as follows from the evident inequality $0 \leq \frac{x}{x+(1-x) \exp (-\alpha g(x))} \leq 1$. Moreover, it is straightforward to see that $x^{*}=0$ and $x^{*}=1$, which correspond to "pure strategies" where "all players play $L$ " and "all players play $R$ ", respectively, are boundary equilibrium points. Interior equilibria exist at any $x^{*}$ such that $g\left(x^{*}\right)=0$, i.e. are characterized by identical payoffs, as we are considering minority games.

In the following, we consider an important class of binary choice games known as minority games characterized by the property that players gain higher payoff when they choose the strategy chosen by the minority of players, i.e. $R(x)$ is higher than $L(x)$ when $x$ is small, whereas $R(x)$ is less than $L(x)$ for values of $x$ close to 1 . A typical example is shown in Figure 1, where the payoff functions are linear, expressed by

$$
R(x)=a x+b ; L(x)=c x+d \text { with } c>a \text { and } 0<\frac{b-d}{c-a}<1 .
$$

For the sake of simplicity we shall use these linear payoff functions in the numerical computations given in this paper, even if the analytical results will be stated for more general nonlinear payoff functions such that:

Assumptions on minority games payoff functions. $R:[0,1] \rightarrow R$ and $L:[0,1] \rightarrow R$ are differentiable functions such $R(0)>L(0), R(1)<$ 
$L(1)$ and they intersect in only one interior point $x^{*} \in(0,1)$ where $R\left(x^{*}\right)=$ $L\left(x^{*}\right)$.

A first consequence of these assumptions is that $g^{\prime}\left(x^{*}\right) \leq 0$. In the following, we shall assume, without loss of generality, that $g^{\prime}\left(x^{*}\right)<0$.

When the evolutionary mechanism (2) is applied under these assumptions, a dynamic behavior characterized by oscillations around the unique Nash equilibrium $x^{*}$ is obtained. These oscillations may converge or not to the Nash equilibrium in the long run according to its stability properties, as stated by the following proposition:

Proposition 1. Under the assumptions on payoff functions stated above, the Nash equilibrium $x^{*}$ is locally asymptotically stable, with oscillatory convergence, provided that

$$
\alpha<\alpha_{f}=-\frac{2}{x^{*}\left(1-x^{*}\right) g^{\prime}\left(x^{*}\right)}
$$

where the derivative $g^{\prime}\left(x^{*}\right)=R^{\prime}(x)-L^{\prime}(x)<0$ is the relative slope of the two payoff curves at their unique intersection $x^{*}$. As the parameter $\alpha$ increases across the threshold value $\alpha_{f}$, then a flip (or period doubling) bifurcation occurs.

Proof. The local stability condition immediately follows from the condition for local asymptotic stability $-1<f^{\prime}\left(x^{*}\right)<1$; see [13]. From (2) this condition becomes

$$
-1<1+\alpha x^{*}\left(1-x^{*}\right) g^{\prime}\left(x^{*}\right)<1 .
$$

Being $g^{\prime}\left(x^{*}\right)<0$ the right inequality is always satisfied, whereas the left one gives (4).

A typical graph of the one-dimensional map (2) with payoff functions (3) is shown in Figure 1b, obtained with parameters $a=-0.5, b=0.5, c=0.8$, $d=0$ and $\alpha=7$. In this case, the Nash equilibrium $x^{*}=\frac{d-b}{a-c}=\frac{5}{13}$ is unstable (being $\alpha>\alpha_{f}=6.5$ ) and the long run dynamics of the minority game settles on a stable cycle of period 2 (shown in the figure) starting from any initial condition $x(0) \in(0,1)$.

As the speed of reaction $\alpha$ is further increased, the well known perioddoubling route to chaos is observed, as shown in the bifurcation diagram 

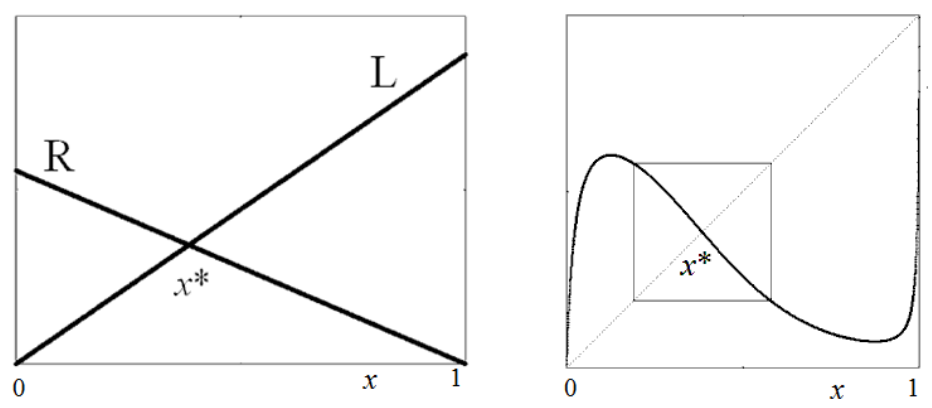

Figure 1: Left: Payoff functions $R(x)$ and $L(x)$ as formalized in (3). Right: Graph of (2), the dynamics of $x(t+1)=f(x(t))$ with $\alpha=7$ and payoff functions (3) with parameters $a=-0.5, b=0.5, c=0.8, d=0$.

of Figure 2. This is a quite expected and well known dynamic behavior in repeated minority games, characterized by contrarians' switches of choices associated with overshooting effects (represented by high values of the speed of reaction $\alpha$ ). In the following, we shall investigate the effects on the stability properties of the Nash equilibrium and, more generally, on the global dynamics of the model, after the introduction of memory effects in this simple evolutionary model proposed.

\section{The two-dimensional model with finite memory of length 1}

The evolutionary models considered in the previous section are based on current payoffs, that is, the players' decisions about the next period strategy choice are based on the knowledge of current payoffs only. A generalization of this assumption consists in replacing the current payoff with a weighted average of it and some of the previously observed ones. In other words, we consider a form of memory in the switching function (2) that describes the number of players that decide to switch their strategy under the evolutionary pressure driven by the payoff observed during the more recent $M$ time periods

$$
U_{R}(t)=\sum_{k=0}^{M} \omega_{k} R(t-k) ; U_{L}(t)=\sum_{k=0}^{M} \omega_{k} L(t-k)
$$

where $M$ is the length of memory and $\omega_{k}$ are the weights, normalized according to $\sum_{k=0}^{M} \omega_{k}=1$. Of course, for $M=0$, the case with no memory is 


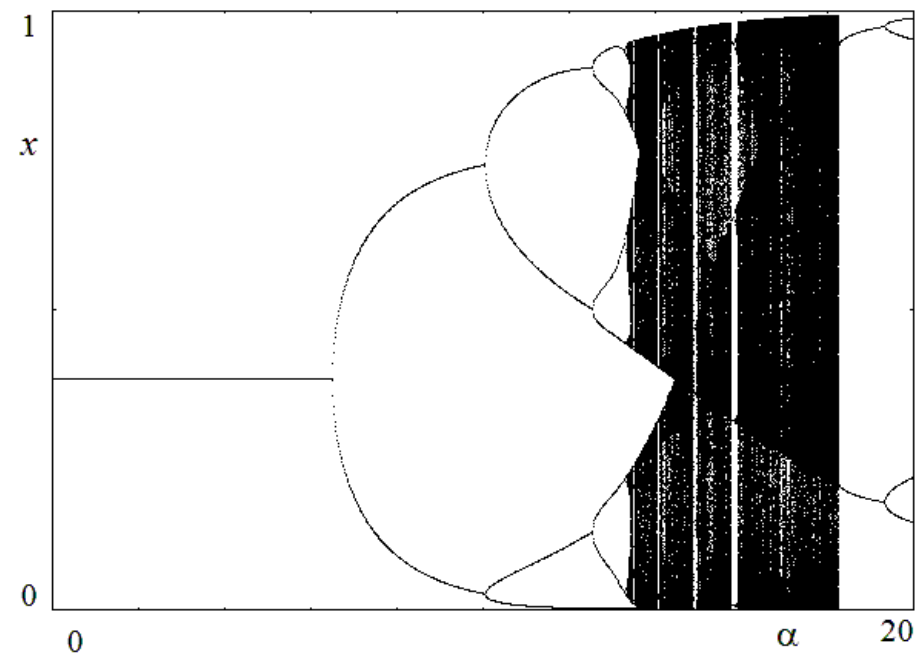

Figure 2: Bifurcation diagram showing $x(t)$, the fraction of agents playing $R$, with bifurcation parameter $\alpha \in[0,20]$ and the other parameters as in Figure 1.

obtained, and, for $M>0$, the distribution of weights can be used to modulate the "shape" of past memory, and how much it differs from the case with no memory. The model (2) with (5), given by

$$
x(t+1)=\frac{x(t)}{x(t)+(1-x(t)) e^{-\alpha\left(U_{R}(t)-U_{L}(t)\right)}}
$$

becomes a difference equation of order $M+1$, equivalent to a $M+1$ dimensional discrete dynamical system. In order to investigate the memory effects and maintain, at the same time, a low dimensionality so that the model is still analytically tractable, we shall consider the case of finite memory of length 1 with weights $\omega_{0}=\omega$ and $\omega_{1}=(1-\omega)$, that is,

$$
\begin{aligned}
& U_{R}(t)=(1-\omega) R(x(t))+\omega R(x(t-1)) \\
& U_{L}(t)=(1-\omega) L(x(t))+\omega L(x(t-1))
\end{aligned}
$$

where $\omega \in[0,1]$. This way, the model reduces to the one without memory, i.e. only current payoff is considered, for $\omega=0$, whereas agents only consider the payoff of the previous period (ignoring the current one) for $\omega=1$. A uniform average of the two payoffs is obtained when $\omega=\frac{1}{2}$, i.e. when considering the arithmetic mean. As usual, after the introduction of the auxiliary 
variable $y(t)=x(t-1)$, the model is expressed by a two dimensional discrete dynamical system

$$
\left\{\begin{array}{l}
x(t+1)=\frac{x(t) e^{\alpha((1-\omega) R(x(t))+\omega R(y(t)))}}{x(t) e^{\alpha((1-\omega) R(x(t))+\omega R(y(t)))}+(1-x(t)) e^{\alpha((1-\omega) L(x(t))+\omega L(y(t)))}} \\
y(t+1)=x(t)
\end{array}\right.
$$

It is easy to check that the equilibria are the same, i.e. $y^{*}=x^{*}$ with $x^{*}=0$, $x^{*}=1$ or $x^{*}$ at the interior intersection $R\left(x^{*}\right)=L\left(x^{*}\right)$. However, the stability conditions are influenced by the "memory parameter" $\omega$, as stated by the following proposition:

Proposition 2. Let $x^{*} \in(0,1)$ be such that $R\left(x^{*}\right)=L\left(x^{*}\right)$ where $R(x)$ and $L(x)$ satisfy the assumptions stated above, and let

$$
\alpha x^{*}\left(1-x^{*}\right) g^{\prime}\left(x^{*}\right)>-4 \text {. }
$$

Then the fixed point $\mathrm{E}=\left(x^{*}, x^{*}\right)$ of $(7)$ is locally asymptotically stable if $\omega_{f}<$ $\omega<\omega_{h}$, with

$$
\omega_{f}=\frac{1}{2}+\frac{1}{\alpha x^{*}\left(1-x^{*}\right) g^{\prime}\left(x^{*}\right)}
$$

and

$$
\omega_{h}=-\frac{1}{\alpha x^{*}\left(1-x^{*}\right) g^{\prime}\left(x^{*}\right)}
$$

If the memory parameter $\omega$ exits the stability interval $\left[\omega_{f}, \omega_{h}\right]$ decreasing through the lower bound $\omega_{f}$, then it loses stability through a flip bifurcation, whereas if $\omega$ exits the stability interval increasing through the upper bound $\omega_{h}$, it loses stability through a supercritical Neimark-Sacker bifurcation.

Proof. The Jacobian matrix of (7) computed at the equilibrium $E$ becomes

$$
J(E)=\left[\begin{array}{cc}
1+\alpha(1-\omega) x^{*}\left(1-x^{*}\right) g^{\prime}\left(x^{*}\right) & \alpha \omega x^{*}\left(1-x^{*}\right) g^{\prime}\left(x^{*}\right) \\
1 & 0
\end{array}\right]
$$

hence $\operatorname{Tr}(E)=1+\alpha(1-\omega) x^{*}\left(1-x^{*}\right) g^{\prime}\left(x^{*}\right)$ and $\operatorname{Det}(E)=-\alpha \omega x^{*}\left(1-x^{*}\right) g^{\prime}\left(x^{*}\right)$ are, respectively, the trace and the determinant of the matrix (11). A sufficient condition for the local asymptotic stability of $E$ is that the eigenvalues of (11), solutions of the characteristic equation $P(z)=z^{2}-\operatorname{Tr}(E) \cdot z+\operatorname{Det}(E)=$ 0 , are located inside the unit circle of the complex plane. A necessary and 
sufficient condition for this is given by the following system of inequalities (known as Schur or Jury's conditions; see e.g. [19], [16], [30])

$$
P(1)=1-\operatorname{Tr}(E)+\operatorname{Det}(E)>0 ; \quad P(-1)=1+\operatorname{Tr}(E)+\operatorname{Det}(E)>0 ; \quad 1-\operatorname{Det}(E)>0
$$

In our case, we have $P(1)=-\alpha x^{*}\left(1-x^{*}\right) g^{\prime}\left(x^{*}\right)>0$ for each set of parameters. Instead, the other two stability conditions become, respectively,

$$
\omega>\frac{2+\alpha x^{*}\left(1-x^{*}\right) g^{\prime}\left(x^{*}\right)}{2 \alpha x^{*}\left(1-x^{*}\right) g^{\prime}\left(x^{*}\right)}=\omega_{f} \text { and } \quad \omega<-\frac{1}{\alpha x^{*}\left(1-x^{*}\right) g^{\prime}\left(x^{*}\right)}=\omega_{h}
$$

where the condition (8) ensures that $\omega_{f}<\omega_{h}$, so that the stability range is not empty. The value of $\omega$ at which $P(-1)$ becomes negative represent a flip (or period doubling) bifurcation value at which an eigenvalue exits the unit circle through the value -1 , and the one at which $1-\operatorname{Det}(E)$ becomes negative represents a Neimark-Sacker bifurcation at which a couple of complex and conjugate eigenvalues exit the unit circle of the complex plane (see e.g. [22] or $[28])$.

It is worth noticing that, as expected, the stability condition

$$
P(-1)=2+\alpha(1-2 \omega) x^{*}\left(1-x^{*}\right) g^{\prime}\left(x^{*}\right)>0,
$$

for $\omega=0$ reduces to the one given in Proposition 1 for the model without memory. However, starting from a value of the speed of reaction $\alpha$ such that $-4<\alpha x^{*}\left(1-x^{*}\right) g^{\prime}\left(x^{*}\right)<-2$, so that the interior equilibrium is unstable when the evolutionary model without memory is considered, according to Proposition 1, and the assumptions of Proposition 2 hold, then the equilibrium gains stability for intermediate values of the memory parameter, i.e. when a weighted average is close to the arithmetic mean. Instead, both for asymmetric averages that give too much weight to the current value or to the previous value, instability prevails.

However, Proposition 2 only concerns the local stability of the unique equilibrium point and gives information neither about its basin of attraction nor about the kind of dynamic behaviors that characterize the long run evolution of the system, when it loses stability through the local bifurcations described in the Proposition. In order to obtain such information, a numerical computation is proposed in the form of a bifurcation diagram obtained with bifurcation parameter $\omega \in[0,1]$, as the one shown in Figure 3, obtained 


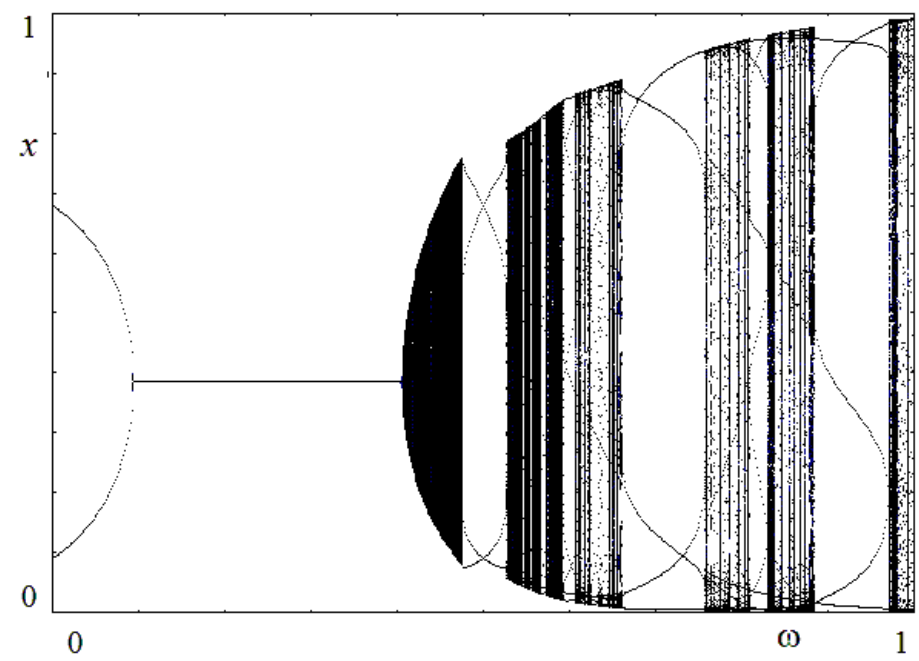

Figure 3: Bifurcation diagram showing $x(t)$, the fraction of agents playing $R$, with bifurcation parameter $\omega \in[0,1]$, speed of reaction $\alpha=0.8$ and the other parameters as the ones used in Figure 1.

with the linear payoff functions (3) and the same parameters as the ones used in Figure 1 (left) and speed of reaction $\alpha=8$. With this set of parameters, we have $\omega_{f}=0.0935$ and $\omega_{h}=0.4065$. As can be seen, the bifurcation diagram in Figure 3 not only confirms the existence of the stability range analytically computed in Proposition 2, but also provides numerical evidence for the supercritical nature of the two local bifurcations, as a stable oscillation of period 2 is obtained as the memory parameter $\omega$ decreases below $\omega_{f}$, and a stable quasi-periodic motion along a closed invariant curve is observed as $\omega$ increases above $\omega_{h}$. Moreover, a representation of the trajectories, the attractors and the basins of attraction in the phase space $(x, y) \in[0,1] \times[0,1]$ clearly show that a unique attractor exists for each constellation of parameters chosen along the bifurcation diagram of Figure 3, and such attractor (that may be a fixed point if $\omega \in\left(\omega_{f}, \omega_{h}\right)$, a stable cycle of period 2 if $\omega<\omega_{f}$ as well as a quasi-periodic, periodic or chaotic attractor for $\left.\omega>\omega_{h}\right)$ is always globally asymptotically stable, in the sense that it attracts almost all the trajectories starting from initial conditions $\left(x_{0}, y_{0}\right) \in(0,1) \times(0,1)$.

However, this is not the end of the story, because for higher values of the speed of reaction $\alpha$, quite different dynamic scenarios are obtained. For example, the bifurcation diagram shown in Figure 4, obtained for $\alpha=11$, 

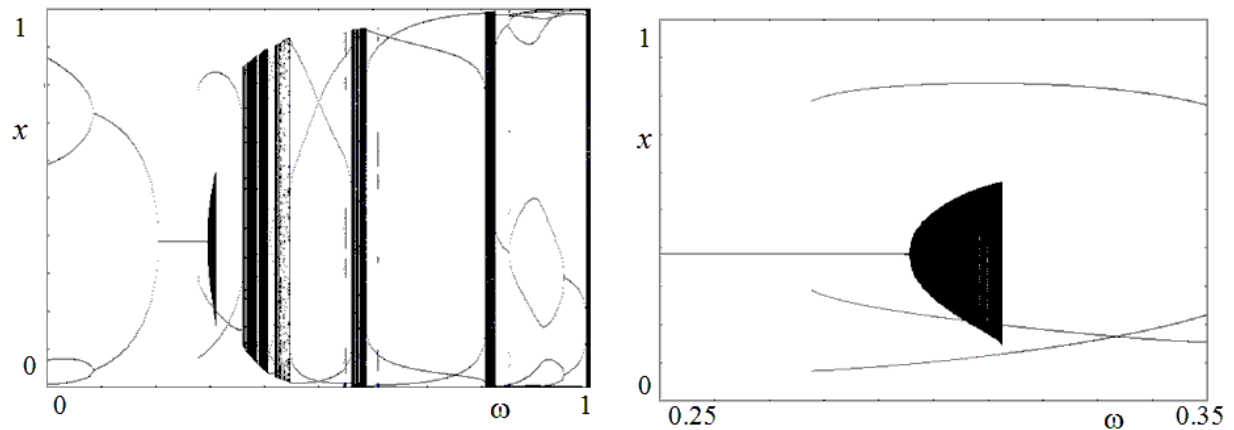

Figure 4: Left: Bifurcation diagram showing $x(t)$, the fraction of agents playing $R$, with bifurcation parameter $\omega$ and $\alpha=11$. Right: enlargement with $\omega \in[0.25,0.35]$.

which gives $\omega_{f}=0.2$ and $\omega_{h}=0.29$, reveals a quite interesting, and unexpected, dynamic scenario. (See in particular the enlargement shown in Figure 4 (right) where the bifurcation parameter $\omega \in[0.25,0.35]$ that reveals a coexistence of two attractors: the stable equilibrium $E$ with a stable cycle of period 3 and then, for $\omega>\omega_{h}$, a stable invariant curve with a stable cycle of period 3.)

It is worth noting that the payoffs along these coexisting attractors are, in general, different. For example, as it concerns the set of parameters used in Figure 5, we have $R(E)=R(E) \simeq 0.3077$, whereas the average population payoff $x R(x)+(1-x) R(x)$, computed in the cycle of period three in the situation of the figure on the left, is 0.1882 . Instead, in the situation of the figure in the center, we have an average population payoff of 0.1755 along the 3 -cycle and 0.2995 along the closed invariant curve, and in the figure on the right it is practically the same along the periodic cycle whereas it is 0.2830 along the invariant curve. It is quite evident that the average population payoff decreases as the system asymptotic dynamics goes far away from the Nash equilibrium.

In this situation of multistability, it is important to study how the coexisting attractors, each with its own basin of attraction, share the phase space on which initial conditions are taken. For example, Figure 5 (left), obtained with $\alpha=11$ and $\omega=0.28<\omega_{h}$, shows the stable equilibrium with its basin of attraction represented by the red region, surrounded by the periodic points of the stable cycle of period 3 with its basin of attraction represented by the darker region. 

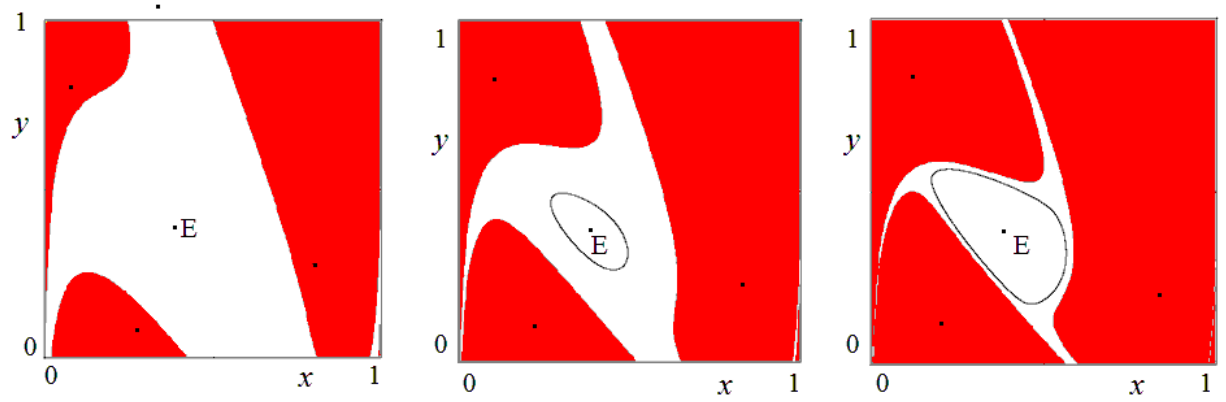

Figure 5: Attractors and basins in the phase space. Left: for $\alpha=11$ and $\omega=0.28$ the stable equilibrium $E$ (white basin) coexist with a stable cycle of period three, the periodic points which are represented by three black dots in the red regions that represent its basin of attraction. Center: $\alpha=11$ and with $\omega=0.3$. Right: $\alpha=11$ and with $\omega=0.31$.

It is worth noting also that such a coexistence could not be predicted by any analytical local analysis of the dynamical system, and, if our analysis were limited to the proof of Proposition 2, together with its immediate numerical confirmation given by the bifurcation diagram of Figure 3, then a quite incomplete, and even misleading, description of the dynamic properties of the evolutionary model considered would be given, just stating that the repeated minority game shows convergence to the unique equilibrium for intermediate values of the memory parameter $\omega$. Instead, after suitable numerical explorations, we can state that, for a wide range of the parameters of the model, the result about local stability of the equilibrium $E$ given in the Proposition 2 only guarantees that the system will converge to such an equilibrium if the initial conditions are taken sufficiently close to it, and larger perturbations will lead the system to exhibit self-sustained bounded oscillations in the long run. (periodic oscillations in the case shown in Figure 4 , but even chaotic for different parameters' constellations).

When comparing our results to [1], where a two-period model is considered as well, some similarities are worth noting. In fact, in their model, the usual complex phenomena that characterize the one-dimensional logistic map disappear when memory weights are close enough to consider only the previously observed state. In addition, in [1], chaos may only occur when weights are on both periods are large enough. This is interesting as, although their map differs from the one we are considering, in both cases the role of having weights on both periods is important. By contrast, in our model we consider adjustment speed as well. This important parameter is related to 
agents' impulsivity [4] and may cause overshooting, with consequences on the multistability of the dynamics. Furthermore, the situation described by the results proved in Proposition 2 has been extensively discussed in the economic literature and has been called sometimes "corridor stability"; see, e.g. [27] or [15]. This stream of literature stresses the fact that nonlinear dynamic models may have the property that small perturbations are recovered as far as they are confined inside the basin of attraction of a locally stable equilibrium, whereas larger perturbations lead to time evolutions that further depart from the equilibrium and go to the coexisting attractor in the long run. The situation may be even more involved when the boundaries that separate the two basins assume a complicated shape (sometimes quite convoluted). Another dynamic scenario is represented in Figure 5 (center) obtained with $\alpha=11$ and with $\omega=0.3>\omega_{h}$. Finally, Figure 5 (right), is obtained with $\alpha=11$ and with $\omega=0.31$. Also in this case, two kinds of asymptotic dynamics can be obtained, given by a quasi-periodic motion along a stable closed invariant curve coexisting with a stable cycle of period 3 , each with its own basin of attraction represented by the white and the red regions, respectively. So, both kinds of long run behaviors are oscillatory, even if the observed oscillations observed are qualitatively different and with different amplitude.

We stress again that such dynamic scenarios, together with their economic consequences, clearly show the importance of a global analysis of nonlinear dynamical systems, which can often be performed only through combining analytical, geometrical and numerical methods. Using only one approach could lead to wrong conclusions about the behavior of the system.

It is also worth remarking that we have not observed such path dependence in the evolutionary model (2) without memory.

\section{Infinite discounted memory}

Following [14], we introduce in the evolutionary model (2) a way to represent players' memory by considering a fitness measure that represents a discounted sum of the payoffs gained along the whole story of the repeated minority game, obtained by taking, at each time step, a convex combination of the current payoff and the fitness measure observed in the previous time period: 


$$
\begin{aligned}
& U_{R}(t)=(1-\omega) R(t)+\omega U_{R}(t-1) \\
& U_{L}(t)=(1-\omega) L(t)+\omega U_{L}(t-1)
\end{aligned}
$$

with $\omega \in[0,1], U_{R}(0)=U_{L}(0)=0$. By backward induction reasoning, it is easy to get

$$
\begin{aligned}
& U_{R}(t)=(1-\omega) \sum_{k=0}^{t-1} \omega^{k} R(t-k)+\omega^{t} U_{R}(0) \\
& U_{L}(t)=(1-\omega) \sum_{k=0}^{t-1} \omega^{k} L(t-k)+\omega^{t} U_{L}(0)
\end{aligned}
$$

which gives the discounted measure of fitness as a weighted sum with exponentially fading weights. Again, the parameter $\omega \in[0,1]$ gives a measure of the memory, as $U_{i}(t)=R(t)$ for $\omega=0$, whereas the arithmetic mean of all the payoffs observed in the past is obtained in the other limiting case, $\omega=1$. If the recursive scheme (13) is plugged into the evolutionary model (2), then we get

$$
\begin{array}{ll}
x(t+1) & =\frac{x(t)}{x(t)+(1-x(t)) \exp \left(-\alpha\left(U_{R}(t)-U_{L}(t)\right)\right)} \\
U_{R}(t+1) & =(1-\omega) R(x(t+1))+\omega U_{R}(t) \\
U_{L}(t+1) & =(1-\omega) L(x(t+1))+\omega U_{L}(t)
\end{array}
$$

and, subtracting the third equation from the second, we get the equivalent 2-dimensional model

$$
\begin{array}{ll}
x(t+1) & =\frac{x(t)}{x(t)+(1-x(t)) \exp (-\alpha \Delta U(t))} \\
\Delta U(t+1) & =(1-\omega) g(x(t+1))+\omega \Delta U(t)
\end{array}
$$

where $g(x)$ is given by $(1)$ and $\Delta U(t)=U_{R}(t)-U_{L}(t)$. The fixed points of this map are given by $E_{0}=(0, g(0)), E_{1}=(1, g(1)), E=\left(x^{*}, 0\right)$ with $x^{*} \in(0,1)$ corresponding with the Nash equilibrium at which $R\left(x^{*}\right)=L\left(x^{*}\right)$.

The following proposition, which should be compared with Propositions 1 and 2, gives the local stability properties of the Nash equilibrium under the assumption of infinite weighted memory.

Proposition 3. If the Nash equilibrium $x^{*}$ is stable under the evolutionary model without memory (2), i.e. $\alpha<\alpha_{f}$, then it is also stable under the model with memory (15). If the Nash equilibrium $x^{*}$ is unstable under the evolutionary model without memory (2), i.e. $\alpha>\alpha_{f}$, then it is stable under the model with memory (15), provided that

$$
\omega>\omega_{s}=\frac{\alpha x^{*}\left(1-x^{*}\right) g^{\prime}\left(x^{*}\right)+2}{\alpha x^{*}\left(1-x^{*}\right) g^{\prime}\left(x^{*}\right)-2} .
$$


Proof. The Jacobian matrix of (15) computed at the equilibrium $E$ becomes

$$
\mathrm{J}(E)=\left[\begin{array}{cc}
1 & \alpha x^{*}\left(1-x^{*}\right) \\
(1-\omega) g^{\prime}\left(x^{*}\right) & \alpha(1-\omega) x^{*}\left(1-x^{*}\right) g^{\prime}\left(x^{*}\right)+\omega
\end{array}\right]
$$

hence $\operatorname{Tr}(E)=1+\alpha(1-\omega) x^{*}\left(1-x^{*}\right) g^{\prime}\left(x^{*}\right)+\omega$ and $\operatorname{Det}(E)=\omega$ are, respectively, the trace and the determinant of the matrix (17). The sufficient conditions for the stability of $E(12)$ in this case become $P(1)=$ $-\alpha(1-\omega) x^{*}\left(1-x^{*}\right) g^{\prime}\left(x^{*}\right)>0$ and $1-\operatorname{Det}(E)=1-\omega>0$ for each set of parameters with $\omega \in[0,1)$, whereas the condition $P(-1)>0$ becomes $2+\omega\left(2-\alpha x^{*}\left(1-x^{*}\right) g^{\prime}\left(x^{*}\right)\right)+\alpha x^{*}\left(1-x^{*}\right) g^{\prime}\left(x^{*}\right)>0$, from which (16) follows. The value of $\omega$ at which $P(-1)$ becomes negative represent a flip (or period doubling) bifurcation value.

It is worth noting that, again, an increase of the memory parameter $\omega$ has a stabilizing effect because, if the Nash equilibrium is stable under the evolutionary dynamics without memory, then it remains stable with memory, whereas an unstable Nash equilibrium under the evolutionary dynamics without memory becomes stable with a sufficiently strong memory. The stability threshold $\omega_{s}$ is an increasing function of $\alpha$ with $\omega_{s}\left(\alpha_{f}\right)=0$ and $\lim _{\alpha \rightarrow \infty} \omega_{s}=1$.

In our model, parameter $\alpha$ controls the propensity to switch choices and directly influences the stability of the dynamics. Nevertheless, having infinite memory plays an important role in the stability and is consistent with previous findings in the economic literature. In particular, in [25] it was proved that, in a non-linear cobweb model, when all past prices are remembered, the dynamics becomes asymptotically stable and chaos cannot occur. Furthermore, when assuming that being able to consider infinitely many periods requires unlimited working memory, our results are consistent with [23] as they showed experimental evidence that limits on the working memory are predictive of more impulsive decisions.

Two typical bifurcation diagrams for increasing values of the memory parameter $\omega$ are shown in Figure 6. The one in the left panel, obtained for $\alpha=10$ and the same payoff function used in all the previous numerical simulations, so that $\omega_{s}=0.211$, reveals that, for $\omega<\omega_{s}$, a stable cycle of period 2, with periodic points, respectively, lower and higher of the Nash value $x^{*}$, characterizes the long run dynamics of the evolutionary minority game. The amplitude of the oscillations decreases as the memory strength increases until the Nash equilibrium becomes stable through a supercritical 

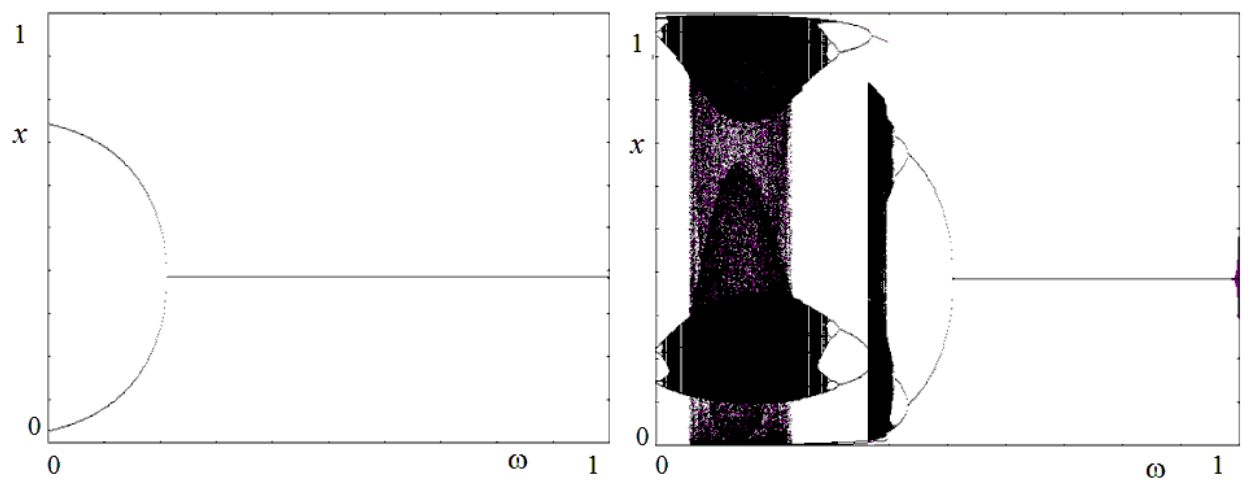

Figure 6: Two bifurcation diagrams showing $x(t)$, the fraction of agents playing $R$, for the model with infinite memory and memory parameter $\omega$ taken as bifurcation parameter, while payoff functions are the same as in all the previous numerical simulations. Left: $\alpha=10$. Right: $\alpha=20$.

flip bifurcation. The bifurcation diagram shown in the right panel of Figure 6 , obtained with $\alpha=20$ (i.e. a higher speed of reaction of the players, a more impulsive behavior when payoff gains are observed), reveals much more complicated dynamic scenarios for low values of the memory parameter, which include periodic and chaotic oscillations with several local and global bifurcations that change the qualitative properties of the attractors, including evident cases of multistability, i.e. coexistence of several attractors each with its own basin of attraction; see e.g. the portion of the bifurcation diagram with $\omega \in(0.3,0.4)$. We do not investigate further such dynamic scenarios, but merely stress that, nevertheless, any complexity disappears as the memory parameter increases beyond the bifurcation value $\omega_{s}$, that with this set of parameters is given by $\omega_{s}=0.506$.

\section{Conclusions}

The effect of memory on minority games is controversial, as proved by [9] and [8]. Furthermore, when, in discrete time periods, agents repeatedly play a minority game, persistent oscillations between the two strategies can be observed.

Dindo in [14], in order to obtain a tractable model, constructs a dynamical system for the minority game and concludes that, in this case, infinite discount memory stabilizes the dynamics. 
In this paper, following the approach suggested in [14], we have proposed some tractable low-dimensional discrete dynamical system, based on exponential replicator dynamics, in order to describe the time evolution of a social system characterized by the interaction of a large number of agents who are facing binary choices. In particular, we used such a framework to analyze the effects of memory on the long run outcomes of a repeatedly played minority game, with two kinds of memory.

The first one - which is more innovative- considers only two states and, in some broad sense, is more similar to the finite states memory considered in [8] and [9], while the second is similar to the one presented in [14].

The results of our analysis confirm the stabilizing effects showed by [14] and may suggest that complex dynamics may be the result of bounds on the human processing capabilities [33]. In fact, with infinite memory, stability is local and there is no evidence of global instability. By contrast, when considering a two-period memory, stability is only local. In fact, by combining analytical, geometrical and numerical methods we were able to find that stability is just local and holds as long as the memory parameter belongs to a neighborhood of $1 / 2$, i.e., as long as the two periods have approximately the same importance.

This result has consequences that go beyond the specific model we were considering. In fact, it shows once more that, when considering nonlinear systems, both local and global analysis are necessary to have better understanding of the system. Indeed, our numerical simulations provide further insight into nonlinear phenomena and the related effects of the presence of memory.

In particular, the model studied in this paper gives us the opportunity to learn an important lesson, because, in some ranges of the parameters such that the equilibrium is locally stable, coexisting periodic and chaotic attractors have been numerically observed, thus giving a strong path dependence. In fact, when the locally stable equilibrium coexists with a different kind of attractor, each with its own basin of attraction, a typical situation of "corridor stability" occurs, as small perturbations (or shocks or historical accident) around the equilibrium are endogenously recovered by the endogenous dynamics of the system, whereas larger perturbations are amplified by the endogenous dynamics, thus leading to a completely different (and non stationary) disequilibrium dynamics, so that only an external control policy can force the system back to the originary equilibrium. These dynamic scenarios clearly show the importance of a global analysis of nonlinear dynam- 
ical systems, which can often be performed only through heuristic methods obtained by a combination of analytical, geometrical and numerical methods. In fact, a study limited to an analytical study of the local stability and bifurcations, based on the linear approximation of the model around the equilibrium points, sometimes may be quite incomplete and even misleading.

In this paper, we focused on minority games. However the mathematical apparatus proposed can be easily extended to several different kinds of binary games. A particularly interesting case that will be examined in the future is given by non-monotonic payoffs functions, characterized by two interior equilibria; see e.g. [31],[21], [5].

Schelling and other authors propose situations where a strategy, say $R$, is convenient for intermediate values of people choosing it, whereas it is not convenient for too low or too high values of $x$.

Of course, extensions are also possible by considering finite memory of length greater than one. However this leads to high dimensional dynamical systems, hence to models that are outside the scope of studies remaining at a level of analytical tractability.

\section{Acknowledgments}

Work developed in the framework of the research project on "Dynamic Models for behavioural economics" financed by DESP-University of Urbino. Ugo Merlone gratefully acknowledges the Department of Economics of Chuo University in Tokyo for funding his participation to the Tokyo NED conference.

\section{Compliance with Ethical Standards}

The authors declare that they have no conflict of interest.

\section{References}

[1] Aicardi, F. and Invernizzi, S. (1992). Memory effects in discrete dynamical systems. International Journal of Bifurcation Chaos, 2(4):815-830.

[2] Allport, F. (1924). Social Psychology. Houghton Mifflin, The Riverside Press, Cambridge, MA. 
[3] Arthur, W. B. (1994). Inductive reasoning and bounded rationality. The American Economic Review, 84:406-411.

[4] Bischi, G. I., Gardini, L., and Merlone, U. (2009). Impulsivity in binary choices and the emergence of periodicity. Discrete Dynamics in Nature and Society, Volume 2009:Article ID 407913, 22 pages doi:10.1155/2009/407913.

[5] Bischi, G. I. and Merlone, U. (2009). Global dynamics in binary choice models with social influence. The Journal of Mathematical Sociology, 33(4):277-302.

[6] Bischi, G. I. and Merlone, U. (2010). Binary choices in small and large groups: A unified model. Physica A: Statistical Mechanics and its Applications, 389(4):843-853.

[7] Cabrales, A. and Sobel, J. (1992). On the limit points of discrete selection dynamics. Journal of Economic Theory, 57:407-419.

[8] Cavagna, A. (1999). Irrelevance of memory in the minority game. Physical Review E, 59(4):3783-3786.

[9] Challet, D. and Marsili, M. (2000). Relevance of memory in minority games. Physical Review E, 62(2):1862-1868.

[10] Challet, D., Marsili, M., and Zhang, Y. (2005). Minority Games. Oxford University Press, Oxford, UK.

[11] Challet, D. and Zhang, Y. (2010). Emergence of cooperation and organization in an evolutionary game. Physica A: Statistical Mechanics and its Applications, 246:407-418.

[12] Cressman, R. and Tao, Y. (2014). The replicator equation and other game dynamics. Proc Natl Acad Sci USA, 111(3):10810-10817.

[13] Devaney, R. L. (1989). An Introduction to Chaotic Dynamical Systems. Perseus Books, Reading, MA, second edition.

[14] Dindo, P. (2005). A tractable evolutionary model for the minority game with asymmetric payoffs. Physica A: Statistical Mechanics and its Applications, 335(4):110-118. 
[15] Dohtani, A., Inaba, T., and Osaka, H. (2007). Time and space in economics. In Asada, T. and Ishikawa, T., editors, Business Cycles Dynamics. Models and Tools, pages 129-143. Springer, New York, NY.

[16] Elaydi, S. (1995). An Introduction to Difference Equations. SpringerVerlag, New York, NY.

[17] Fazeli, A. and Jadbabaie, A. (2012). Duopoly pricing game in networks with local coordination effects. pages 74-79, Maui, Hawaii.

[18] Franke, R. (2003). Reinforcement learning in the El Farol model. Journal of Economic Behavior \&3 Organization, 51:367-388.

[19] Gandolfo, G. (2010). Economic Dynamics. Springer-Verlag, New York, NY.

[20] Granovetter, M. (1978). Threshold models of collective behavior. The American Journal of Sociology, 83(1):1420-1443.

[21] Granovetter, M. and Soong, R. (1983). Threshold models of diffusion and collective behavior. Journal of Mathematical Sociology, 9(3):165-179.

[22] Guckenheimer, J. and Holmes, P. (1983). Nonlinear Oscillations, Dynamical Systems, and Bifurcations of Vector Fields. Springer-Verlag, New York, NY.

[23] Hinson, J. M., Jameson, T. L., and Whitney, P. (2003). Impulsive decision making and working memory. Journal of Experimental Psychology Learning Memory and Cognition, 29:298-306.

[24] Hofbauer, J. and Sigmund, K. (2003). Evolutionary game dynamics. Bulletin of The American Mathematical Society, 40(4):479-519.

[25] Holmes, J. and Manning, R. (1988). Memory and market stability: the case of the cobweb. Economic Letters, 28:1-7.

[26] Hovi, J. (1986). Binary games as models of public goods provision. Scandinavian Political Studies, 9(4):337-360.

[27] Leijonhufvud, A. (1973). Effective demand failures. The Swedish Journal of Economics, 75(1):27-48. 
[28] Lorenz, H. (1993). Nonlinear Dynamical Economics and Chaotic Motion. Springer-Verlag, New York, NY, second edition.

[29] Mahajan, V., Muller, E., and Bass, F. M. (1990). New product diffusion models in marketing: A review and directions for research. Journal of Marketing, 54(1):1-26.

[30] Medio, A. and Lines, M. (2001). Nonlinear Dynamics. Cambridge University Press, Cambridge, UK.

[31] Schelling, T. C. (1973). Hockey helmets, concealed weapons, and daylight saving: A study of binary choices with externalities. The Journal of Conflict Resolution, 17(3):381-428.

[32] Schelling, T. C. (1978). Micromotives and Macrobehavior. W. W. Norton, New York, NY.

[33] Simon, H. A. (1990). Bounded rationality. In Eatwell, J., Milgate, M., and Newman, P., editors, Utility and Probability, pages 15-18. Palgrave Macmillan UK, London.

[34] Stahl, D. O. and Haruvy, E. (2008). Level- $n$ bounded rationality and dominated strategies in normal-form games. Journal of Economic Behavior 83 Organization, 66:226-232.

[35] van Ginneken, J. (2003). Collective Behavior Public Opinion. Lawrence Erlbaum Associates, Mahwah, NJ. 